\title{
A Surgical Procedure and Prognosis for Gallbladder Carcinoma According to the Extent of the Tumor Invasion-A Retrospective Case Series Study at a Japanese Hospital
}

\section{Akitsugu Makino', Hiroshi Nakagomi ${ }^{*}$, Atsushi Takano', Masahiro Maruyama1, Kazunori Takahashi', Yuko Nakayama', Masayuki Inoue', Kazushige Furuya', Hidemitsu Sugai', Masao Hada', Yoshiaki Miyasaka1 and Toshio Oyama ${ }^{2}$}

\author{
${ }^{1}$ Department of Surgery, Yamanashi Prefectural central Hospital, Japan \\ ${ }^{2}$ Department of Pathology, Yamanashi Prefectural central Hospital, Japan
}

*Corresponding author: Hiroshi Nakagomi, Department of Surgery, Yamanashi Prefectural Central Hospital, Fujimi1-1-1, Kofu, Yamanashi, Japan, Tel: +81-55-253-7111, Fax: +81-55-253-8011, E-mail: h-nakagomi@ych.pref.yamanashi.jp

\begin{abstract}
Purpose: To analyze the prognostic effects of our selecting surgical procedures for gallbladder carcinoma based on the extent of tumor invasion.

Methods: We reviewed 35 patients with gallbladder carcinoma who underwent the surgical treatment in our hospital. We divided the patients into three groups ( 8 with T1, 15 with T2, and 12 patients with T3) according to the pathological extent of the tumor invasion. Results: Although 8 patients with T1 group had been achieved curative operation (R0), 2 patients developed an unexpected recurrence regardless the kinds of surgical procedures.

Regarding the 15 patients in T2, the prognosis of 11 patients with T2n- was satisfactory. Of the 4 patients with $T 2 n+$, only 3 patients who underwent an extent cholecystectomy (ExC) and lymph node dissection (LND) with Extra Hepatic Bile Duct Resection (EHBDR) had no recurrence.

Of the 12 patients in T3, 5 patients were under the limited operation. Although, remaining 6 patients were expected to achieve curative operation (RO), all patients died.

Conclusions: Our data indicated ExC+LND with the EHBDR achieved survival cases in T1/T2 group who had an expected LN metastasis. However, all patients in the T3 group died from distant metastases and peritoneal disseminations, suggesting that the surgical treatment is insufficient to overcome T3 gallbladder
\end{abstract} carcinomas.

\section{Keywords}

Gallbladder carcinoma, Extra hepatic bile duct resection, Lymph node dissection, Survival

\section{Abbreviations}

OC: Open Cholecystectomy, ExC: Extent Cholecystectomy, LND Lymph Node Dissection, EHBDR: Extra hepatic Bile Duct resection, PD: Pancreaticoduodenectomy

\section{Introduction}

The incidence of gall bladder and bile duct cancer in Japan was approximately 21,000 in 2008 , and accounted for $2.8 \%$ of all cancer patients and 18,000 patients died annually [1]. The prognosis for gallbladder and bile duct carcinoma is poor. The complete resection (R0) surgical procedure is considered to be the only way to cure the patient [2].

Gallbladder Carcinoma (GC) has a particularly low incidence, and as a result, few randomized control trials have been conducted to determine the optimal surgical procedure. There is evidence that the optimal treatment modality is a simple cholecystectomy for a GC with a mucosal invasion (T1a) [3,4]. Even still, there are many controversial issues concerning the surgical treatments for GC that invade beyond the muscular is (>T1b) $[5,6]$. The necessity of an extra hepatic bile duct resection (EHBDR) with a lymph node dissection for the treatment of a T2 GC has been consistently discussed $[7,8]$. However, we have no clear evidence to show the effectiveness of the EHBDR procedure. Furthermore, the need for an extended and radical operation for a T3 level invasion with an extensive GC has also been discussed $[9,10]$.

We retrospectively analyzed 35 patients with GC who underwent surgical treatment in our hospital. We herein describe our selection of surgical procedure according to the extent of the tumor invasion (T1, T2, and T3) and clinical course of the patients in each group.

\section{Materials and Methods}

Eighty six patients with GC had been listed in the cancer registration in our hospital between the time period of January, 2007 and December, 2013. Among these, 35 (16 of male and 19 female) patients underwent the surgical treatment. The mean age of the patients was $73 \pm 8$ years of age (range, 54-88). We reviewed the patients' clinical records of the surgical procedure, including the extent of lymph node dissection, the survival data and the pathological

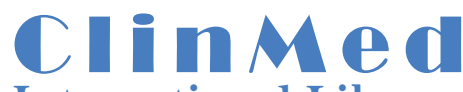

International Library
Citation: Makino A, Nakagomi H, Takano A, Maruyama M, Takahashi K, et al. (2015) A Surgical Procedure and Prognosis for Gallbladder Carcinoma According to the Extent of the Tumor Invasion -A Retrospective Case Series Study at a Japanese Hospital. Int J Surg Res Pract 2:025

Received: July 06, 2015: Accepted: August 11, 2015: Published: August 14, 2015 Copyright: (c) 2015 Makino A. This is an open-access article distributed under the terms of the Creative Commons Attribution License, which permits unrestricted use, distribution, and reproduction in any medium, provided the original author and source are credited. 
findings, including the extent of tumor invasion, vascular invasion, and the number of lymph node metastasis, the histological grade and final stage according to the UICC TNM criteria.

The survival time was analyzed by the Kaplan-Meier method and the statistical significances were tested with the log-rank test using the SPSS ver.21 statistical software.

Patients were divided into three groups: 1) T1; the tumor invaded the mucosa (T1a) and muscular is (T1b), 2) T2; the tumor invaded the peri muscular connective tissue without extension beyond the serosa and 3) T3; the tumor perforated the serosa or directly invaded other organs, including liver and common bile duct.

Eight patients in the T1 group, 15 patients in the T2 group, and 12 patients in the T3 group with GC were analyzed for the surgical procedures and their prognosis.

\section{Results}

Mean survival time of T1, T2 and T3 GC was $62 \pm 0,48 \pm 6$ and 13 \pm 3 , respectively. And significant differences were observed between T1-T3 $(\mathrm{p}=0.00)$, and T2-T3 $(\mathrm{p}=0.00)$ on log-rank test (Figure 1). The 1,3 and 5 year survival rates of all $(n=35)$ patients with $\mathrm{GC}$ were 72 , 49 , and $42 \%$, respectively.

All of the selected factors such as the extent of tumor invasion (pT), vascular invasion, lymph node metastasis, histological grade and the f-Stage according to the UICC TNM classification, significantly correlated with mean survival time (MST) of patients with GC (Table 1). The extent of the tumor invasion is one of the most important prognostic factors which could be predicted by preoperative evaluations.

Among the eight patients with $\mathrm{T} 1$ (T1a;6, T1b;2), 4 patients underwent an Open Cholecystectomy (OC) only, 2 patients underwent

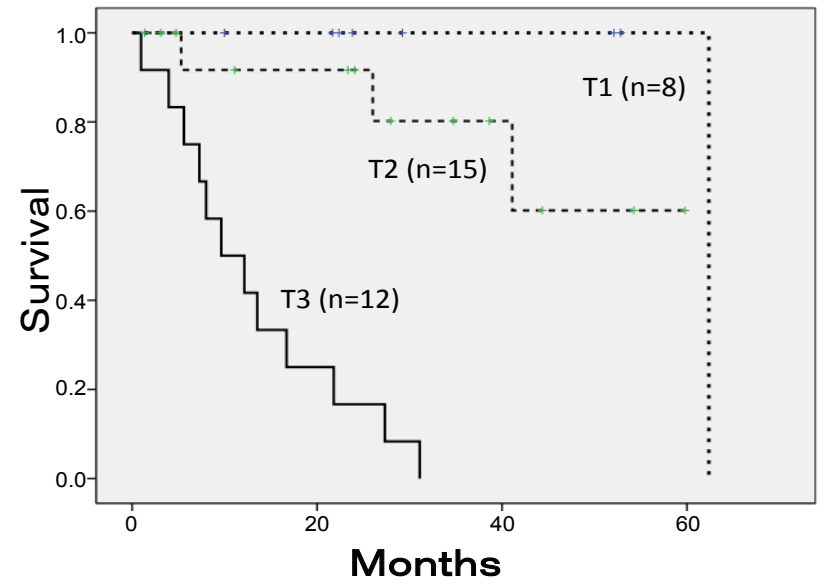

Figure 1: Survival Curve of 35 patients with gallbladder carcinoma who underwent surgery using Kaplan-Meier methods. Mean survival time of T1, T2 and T3 GC was $62 \pm 0,48 \pm 6$ and $13 \pm 3$ months, respectively. Statistical significance with log-rank test are T1-T2 $(p=0.17)$, T1-T3 $(p=0.00)$, and T2T3 $(p=0.00)$ an OC or an extent cholecystectomy (ExC; cholecystectomy with wedge resection or a segmentectomy of the liver) with a Lymph Node Dissection (LND) and 2 patients underwent a (ExC) and a LND with an Extra Hepatic Bile Duct Resection (EHBDR) (Table 2). Two of 8 patients with T1, developed an unexpected recurrence regardless the kinds of surgical procedure. One patient with T1b developed a regional lymph node metastasis despite the diagnosis of $\mathrm{n} 0$ in the dissected LN. Another patient with a T1a GC whose nuclear grade was G3 developed an unexpected LN metastasis.

Of the 15 patients with the $\mathrm{T} 2 \mathrm{GC}$, the prognosis of 11 of the patients with T2n-(f stage 2 ) was satisfactory (Table 2,3). One patient who received a Laparoscopic Cholecystectomy (LC) developed a peritoneal metastasis and died. The other 10 patients had no recurrence of disease, and of these patients, 1 patient underwent an OC only, 4 patients received an $\mathrm{ExC}+\mathrm{LND}$, and 4 patients received an ExCH+LND with an EHBDR.

Of the 4 patients with $\mathrm{T} 2 \mathrm{n}+$ (f stage; $3 \mathrm{~b}$ ), 3 patients who underwent an ExC+LND with an EHBDR had no recurrence and, their lymph node metastasis was limited to one lymph node. One patient who underwent a Pancreaticoduodenectomy (PD) and who had 4 metastasis of 12 dissected lymph nodes, developed a peritoneal and a regional lymph node metastasis 1 year later, and died 2 years after the operation.

Of the 12 patients with a T3 GC, 3 patients underwent an ExC only and 2 patients underwent an ExC+LND as a limited operation (Table 4). Although, the 6 patients who underwent an ExC+LND with an EHBDR, were expected to achieve a curative operation (R0), all of these patients died. Most of the patients developed liver and peritoneal metastases.

\section{Discussion}

The screening program for hepato-biliary morbidity using

Table 1: Correlation between Mean Survival Time (MST) and pathological factors p- values were analyzed by log-rank test

\begin{tabular}{|c|c|c|c|c|}
\hline Pathological Factors & $\mathbf{n}$ & MST & $95 \% \mathrm{Cl}$ & P-values \\
\hline \multicolumn{5}{|l|}{ Primary tumor } \\
\hline $\mathrm{T} 1$ & 8 & 62 & na & $\mathrm{p}=0.03(\mathrm{~T} 1 \mathrm{vs} \mathrm{T} 2)$ \\
\hline T2 & 15 & 35 & $(25 \sim 47)$ & $p=0.01($ T2 vs T3) \\
\hline T3 & 12 & 14 & $(4 \sim 23)$ & $p=0.02(T 1$ vs T3) \\
\hline \multicolumn{5}{|l|}{ Vascular invasion } \\
\hline no & 15 & 51 & $(38 \sim 64)$ & \multirow{2}{*}{$p=0.01$} \\
\hline yes & 20 & 27 & $(16 \sim 37)$ & \\
\hline \multicolumn{5}{|l|}{ Lymph node metastasis } \\
\hline no & 20 & 51 & $(40 \sim 62)$ & \multirow{2}{*}{$p=0.00$} \\
\hline yes & 15 & 17 & $(12 \sim 24)$ & \\
\hline \multicolumn{5}{|l|}{ Histological grade } \\
\hline 1 & 22 & 50 & $(40 \sim 60)$ & $p=0.03(G 1$ vs G2) \\
\hline 2 & 5 & 11 & $(5 \sim 16)$ & $p=0.01(G 2$ vs G3) \\
\hline 3 & 8 & 14 & $(7 \sim 47)$ & $p=0.02(G 1$ vs G3) \\
\hline \multicolumn{5}{|l|}{ f Stage } \\
\hline 1 & 8 & 57 & $(44 \sim 70)$ & $\mathrm{p}=0.14(\mathrm{~S} 1 \mathrm{vs} \mathrm{S} 2)$ \\
\hline 2 & 10 & 39 & $(26 \sim 52)$ & $p=0.00(S 2$ vs S3) \\
\hline 3 & 17 & 18 & $(11 \sim 24)$ & $\mathrm{p}=0.06$ (S1 vs S3) \\
\hline
\end{tabular}

Table 2: The Characteristics of Patients with T1 Gallbladder Carcinoma

\begin{tabular}{|c|c|c|c|c|c|c|c|c|c|c|c|}
\hline No & Age & Sex & Surgery & $\begin{array}{l}\text { Extent of Tumor } \\
\text { Invasion }\end{array}$ & $\begin{array}{l}\text { Vascular } \\
\text { invasion }\end{array}$ & $\begin{array}{l}\text { lymphnode } \\
\text { metastasin }\end{array}$ & $\begin{array}{l}\text { Histological } \\
\text { Grade }\end{array}$ & f Stage & Reccurence & $\begin{array}{c}\text { No. of } \\
\text { Metastatic LN }\end{array}$ & $\begin{array}{c}\text { No. of } \\
\text { Dissected LN }\end{array}$ \\
\hline 1 & 62 & $\mathrm{~m}$ & OC & $\mathrm{m}$ & 0 & 0 & 1 & 1 & none & na & 0 \\
\hline 2 & 80 & $f$ & OC & $\mathrm{m}$ & 0 & 0 & 1 & 1 & none & na & 0 \\
\hline 4 & 82 & $\mathrm{~m}$ & OC & $\mathrm{m}$ & 0 & 0 & 1 & 1 & none & na & 0 \\
\hline 5 & 65 & $\mathrm{~m}$ & $\mathrm{OC}+\mathrm{LND}$ & $\mathrm{mp}$ & 0 & 0 & 1 & 1 & regional LN & 0 & 5 \\
\hline 6 & 70 & $f$ & ExC+LND & $\mathrm{mp}$ & 0 & 0 & 1 & 1 & none & 0 & 4 \\
\hline 8 & 72 & $\mathrm{~m}$ & ExC+LND+EHBDR & $\mathrm{m}$ & 1 & 1 & 3 & 3 & sc LN & 2 & 4 \\
\hline
\end{tabular}

OC: Open Cholecystectomy, LND: Lymph Node Dissection, ExC: Extended Cholecystectomy, EHBDR: Extrahepatic Bile Duct Resection, m: mucosa, mp: musculer propria, na: not aplicable, sc: supraclavicular 
Table 3: The Characteristics of Patients with T2 Gallbladder Carcinoma

\begin{tabular}{|c|c|c|c|c|c|c|c|c|c|}
\hline No & Age & Sex & Surgery & $\begin{array}{l}\text { Extent of Tumor } \\
\text { Invasion }\end{array}$ & Histological Grade & f Stage & Reccurence Sites & No. of Metastatic LN & No. of Dissected LN \\
\hline 1 & 57 & $f$ & LC & ss & 1 & 1 & peritoneum & na & 0 \\
\hline 2 & 88 & $f$ & $\mathrm{OL}$ & ss & 2 & 2 & none & na & 0 \\
\hline 3 & 72 & $f$ & ExC+LND & ss & 1 & 2 & none & 0 & 6 \\
\hline 4 & 71 & $f$ & ExC+LND & ss & 2 & 2 & none & 0 & 13 \\
\hline 5 & 82 & $\mathrm{~m}$ & ExC+LND & ss & 1 & 2 & none & 0 & 4 \\
\hline 6 & 77 & $f$ & ExC+LND & ss & 1 & 2 & none & 0 & 4 \\
\hline 7 & 54 & $f$ & ExC+LND+EHBDR & ss & 1 & 2 & none & 0 & 14 \\
\hline 8 & 86 & $\mathrm{~m}$ & ExC+LND+EHBDR & ss & 3 & 2 & none & 0 & 0 \\
\hline 9 & 69 & $\mathrm{~m}$ & ExC+LND+EHBDR & ss & 1 & 2 & none & 0 & 10 \\
\hline 10 & 75 & $\mathrm{~m}$ & ExC+LND+EHBDR & ss & 1 & 2 & none & 0 & 7 \\
\hline 11 & 74 & $\mathrm{~m}$ & ExC+LND+EHBDR & ss & 1 & 2 & none & 0 & 17 \\
\hline 12 & 79 & $\mathrm{~m}$ & ExC+LND+EHBDR & ss & 1 & $3 b$ & none & 1 & 8 \\
\hline 13 & 75 & $\mathrm{~m}$ & ExC+LND+EHBDR & ss & 1 & $3 b$ & none & 1 & 9 \\
\hline 14 & 76 & $\mathrm{~m}$ & ExC+LND+EHBDR & $\mathrm{s}$ & 1 & $3 b$ & none & 1 & 3 \\
\hline 15 & 82 & $f$ & ExC+PD & ss & 1 & $3 b$ & liver, peritoneum & 4 & 12 \\
\hline
\end{tabular}

LC: Laparoscopic Cholecustectomy, OC: Open Cholecystectomy, LND: Lymph Node Dissection, ExC: Extended Cholecystectomy, EHBDR: Extrahepatic Bile Duct Resection, s: serosa, ss: subserosa

Table 4: The Characteristics of Patients with T3 Gallbladder Carcinoma

\begin{tabular}{|c|c|c|c|c|c|c|c|c|c|}
\hline No & Age & Sex & Surgery & $\begin{array}{l}\text { Extent of Tumor } \\
\text { Invasion }\end{array}$ & Histological Grade & f Stage & Reccurence Sites & No. of Metastatic LN & No. of Dissected LN \\
\hline 1 & 73 & $\mathrm{~m}$ & ExC+LND & si & 2 & $3 a$ & regional LN & na & 0 \\
\hline 2 & 78 & $f$ & $\mathrm{ExC}+\mathrm{LND}+\mathrm{EHBDR}$ & ss + liver & 3 & $3 b$ & regional LN & 0 & 12 \\
\hline 3 & 79 & $f$ & ExC & ss + liver & 2 & $3 b$ & liver & 1 & 1 \\
\hline 4 & 65 & $\mathrm{~m}$ & ExC & se & 3 & $3 a$ & $p+$ regional LN & 1 & 1 \\
\hline 5 & 75 & $f$ & $\mathrm{ExC}+\mathrm{LND}+\mathrm{EHBDR}$ & se & 2 & $3 a$ & liver + regional LN & 1 & 10 \\
\hline 6 & 70 & $\mathrm{~m}$ & ExC+LND+EHBDR & $\mathrm{se}+\mathrm{CBD}$ & 1 & $3 a$ & $p+$ regional LN & 8 & 11 \\
\hline 8 & 66 & $f$ & $\mathrm{ExC}+\mathrm{LND}+\mathrm{EHBDR}$ & ss + liver & 3 & $3 b$ & $\mathrm{p}$ & 1 & 1 \\
\hline 9 & 85 & $\mathrm{~m}$ & ExC+LND+EHBDR & se + liver & 1 & $3 b$ & $p+$ regional LN & 1 & 8 \\
\hline 10 & 69 & $f$ & ExC+LND+EHBDR & ss + liver & 3 & $3 b$ & liver & 3 & 3 \\
\hline 11 & 75 & $f$ & ExC & si + liver & 3 & $3 b$ & unknown & 2 & 3 \\
\hline 12 & 77 & $f$ & ExC+LND & se + liver & 3 & $3 b$ & liver & 1 & 5 \\
\hline
\end{tabular}

LND: Lymph Node Dissection, ExC: Extended Cholecystectomy, EHBDR: Extrahepatic Bile Duct Resection, PD: Pancreoticoduodenectomy, CBD: Common Bile Duct, p: peritoneal dissemination

abdominal ultrasound has been widespread in Japan for several decades [11]. Consequently, the frequency of incidental gallbladder carcinoma that is being diagnosed during laparoscopic surgery for benign diseases is increasing $[12,13]$. However, the prognosis for patients with GC has not been improved $[14,15]$.

There are evidences that the optimal treatment modality is a simple cholecystectomy for a GC with mucosal invasion (T1a) $[3,4]$. However, there are still many controversial issues concerning the surgical treatment of GC that invades beyond the muscularis (>T1b) $[5,6]$. Our selection of surgical procedure for T1 GC was in accordance to the standard surgery mentioned above. However, an extraordinary recurrence happened in 2 of the 8 cases (25\%). A LND should be performed for the cases that are expected to be associated with invasion of the muscularis.

Lymph node metastasis is a well- known prognostic indicator, and its incidence varies depending on the depth of the mural invasion and the depth is scored as follows: pT1a; $0-2.5 \%, \mathrm{pT} 1 \mathrm{~b} ; 5-16 \%, \mathrm{pT} 2$; $9-30 \%$, T3; $39-72 \%$, and T4; $67-80 \%$ [15]. We should be aware of the necessity for a lymph node dissection; however, there is no consensus on the optimal extent of the lymph node dissection for GC surgery.

The clinical significance of the extra hepatic bile duct resection (EHBDR) in radical surgery has been extensively discussed. However, it was subsequently found that an EHBDR is not associated with an increased in survival $[16,17]$. An EHBDR should be selectivity performed in specific types of cases, such as in a GC with an extra hepatic bile duct invasion. A multi-centric questionnaire survey by the Japanese Society of Biliary Surgery [18] concluded that EHBDR may be unnecessary in advanced GC without a direct infiltration of the hepato duodenal ligament and the cystic duct, even in the T2 gallbladder carcinoma with lymph node metastasis.
In our experiments, we achieved good survival in the T2n0 cases with an ExC and a LND with and without the EHBD resection. However, complete resection (R0) could not be achieved by a LND without the EHBDR in one patient with T1 GC. In contrast, we had 3 cases of survival with T2n+ by an ExC+LND with an EHBDR. We decided to select an ExC+LND with an EHBDR for the patients with $\mathrm{T} 1 / \mathrm{T} 2$ and an expected LN metastasis.

Patients with a T3/T4 GC rarely receive the benefit of an extended radical resection because the risk of having a lymph node metastasis beyond the hepatoduodenal ligament, and the latent liver or peritoneal metastasis is high [19]. Most of the T3 patients in this paper developed distant metastases and peritoneal disseminations, which indicates that a more extended radical resection does not improve the prognosis of T3 GC.

We understood through this investigation, that the most important fact to improve the prognosis of a patient with GC is early detection. The screening program to diagnose T1 GC needs to be investigated.

Another important issue to consider is the nonsurgical treatment for GC. Recently, the effect of chemotherapies such as a fluoro pyrimidine containing regimen, gemcitabine, or a combination of gemcitabine and cisplatin has raised concerns. We do not have any established data that indicates the survival benefit of adjuvant chemotherapies yet. However physicians should consider administering these anti-cancer drugs for patients diagnosed to have $\mathrm{T} 3 / \mathrm{T} 4$ or $\mathrm{T} 2$ with lymphno metastasis or any $\mathrm{T}$ with positive margin

\section{Acknowledgments}

We thank all of the members of cancer registration for their helpful assistance. 


\section{References}

1. Cancer mortality rate (2012).

2. Jayaraman S, Jarnagin WR (2010) Management of gallbladder cancer Gastroenterol Clin North Am 39: 331-342, x.

3. You DD, Lee HG, Paik KY, Heo JS, Choi SH, et al. (2008) What is an adequate extent of resection for T1 gallbladder cancers? Ann Surg 247: 835-838.

4. Shirai Y, Yoshida K, Tsukada K, Muto T, Watanabe H (1992) Early carcinoma of the gallbladder. Eur J Surg 158: 545-548.

5. Wakai T, Shirai Y, Yokoyama N, Nagakura S, Watanabe H, et al. (2001) Early gallbladder carcinoma does not warrant radical resection. $\mathrm{Br} \mathrm{J}$ Surg 88: $675-678$

6. Muratore A, Polastri R, Bouzari H, Vergara V, Capussotti L (2000) Radical surgery for gallbladder cancer: a worthwhile operation? Eur J Surg Oncol 26: $160-163$.

7. Aramaki M, Matsumoto T, Shibata K, Himeno Y, Yada K, et al. (2004) Factors influencing recurrence after surgical treatment for T2 gallbladder carcinoma. Hepato gastroenterology 51: 1609-1611.

8. Wise PE, Shi YY, Washington MK, Chapman WC, Wright JK, et al. (2001) Radical resection improves survival for patients with pT2 gallbladder carcinoma. Am Surg 67: 1041-1047.

9. Cubertafond P, Mathonnet M, Gainant A, Launois B (1999) Radical surgery for gallbladder cancer. Results of the French Surgical Association Survey. Hepatogastroenterology 46: 1567-1571.

10. Nimura Y, Hayakawa N, Kamiya J, Maeda S, Kondo S, et al. (1991) Hepatopancreatoduodenectomy for advanced carcinoma of the biliary tract. Hepatogastroenterology 38: 170-175.

11. Okamoto M, Okamoto H, Kitahara F, Kobayashi K, Karikome K, et al (1999) Ultrasonographic evidence of association of polyps and stones with gallbladder cancer. Am J Gastroenterol 94: 446-450.
12. Cavallaro A, Piccolo G, Panebianco V, Lo Menzo E, Berretta M, et al. (2012) Incidental gallbladder cancer during laparoscopic cholecystectomy: managing an unexpected finding. World J Gastroenterol 18: 4019-4027.

13. Fuks D, Regimbeau JM, Le Treut YP, Bachellier P, Raventos A, et al. (2011) Incidental gallbladder cancer by the AFC-GBC-2009 Study Group. World J Surg 35: 1887-1897.

14. Witjes CD, van den Akker SA, Visser O, Karim-Kos HE, de Vries E, et al. (2012) Gallbladder cancer in the Netherlands: incidence, treatment and survival patterns since 1989. Dig Surg 29: 92-98.

15. Lee SE, Kim KS, Kim WB, Kim IG, Nah YW, et al. (2014) Practical guidelines for the surgical treatment of gallbladder cancer. J Korean Med Sci 29: 13331340.

16. Yamaguchi K, Chijiiwa K, Saiki S, Nishihara K, Takashima M, et al. (1997) Retrospective analysis of 70 operations for gallbladder carcinoma. Br J Surg 84: 200-204.

17. Kosuge T, Sano K, Shimada K, Yamamoto J, Yamasaki S, et al. (1999) Should the bile duct be preserved or removed in radical surgery for gallbladder cancer? Hepatogastroenterology 46: 2133-2137.

18. Araida T, Higuchi R, Hamano M, Kodera Y, Takeshita N, et al. (2009) Should the extrahepatic bile duct be resected or preserved in $\mathrm{R} 0$ radical surgery for advanced gallbladder carcinoma? Results of a Japanese Society of Biliary Surgery Survey: a multicenter study. Surg Today 39: 770-779.

19. Kai M, Chijiiwa K, Ohuchida J, Nagano M, Hiyoshi M, et al. (2007) A curative resection improves the postoperative survival rate even in patients with advanced gallbladder carcinoma. J Gastrointest Surg 11: 1025-1032. 